\title{
Simple Model of Transformation of a Crystal Structures
}

\author{
B. I. Lev ${ }^{1,2}$ \\ ${ }^{1}$ Bogolyubov Institute for Theoretical Physics of the NAS of Ukraine, Kyiv, Ukraine \\ ${ }^{2}$ Department of Physics, Chungnam National University, Daejeon, Korea \\ Email: blev@bitp.kiev.ua
}

How to cite this paper: Lev, B.I. (2016) Simple Model of Transformation of a Crystal Structures. Journal of Modern Physics, 7 , 2366-2373.

http://dx.doi.org/10.4236/jmp.2016.716205

Received: November 13, 2016

Accepted: December 23, 2016

Published: December 26, 2016

Copyright (๑) 2016 by author and Scientific Research Publishing Inc. This work is licensed under the Creative Commons Attribution International License (CC BY 4.0).

http://creativecommons.org/licenses/by/4.0/

\begin{abstract}
A simple model of the closely packed structure for system of hard-sphere particles interacting via the long-range Newtonian type attraction is suggested. Based on density functional theory, the exact equation of state is obtained and the mutual transformations of the crystal structures in such systems are studied. The description takes into account the fact impossibility of hard-sphere particles which have the same spatial occupation place.
\end{abstract}

\section{Keywords}

Density Functional Theory, Transformation of Structures, Close-Packing Structure

The statistical and thermodynamic description of a system of interacting particles is one of the basic problems in condensed matter physics. In this direction, significant successes have been achieved [1]-[13]. The special method used in these researches is based on a density functional theory [1]-[13]. Within the framework of this approach, the phase transitions in a system of interacting particles from a gas phase to liquid and also from liquid to solid are described [14]-[23]. Various models of the phase formation and separation in such systems are considered depending on the strength and nature of inter-particle interaction [1] [2] [3].

The classical density-functional theory has become increasingly popular in the study of liquid-solid transition [1] [2] [3] [4]. This is partially due to the success in providing an accurate description of the fluid to close-packed solid transition for a system of hard spheres [2] [3]. On the basis of these results, it seems that the density-functional theory works well at least for systems of monatomic particles with hard interactions for which the equilibrium solid phase is close packed [2] [3] [4]. The question is whether the 
success of the density-functional theories with hard interactions extends to systems with softer interactions where the thermodynamically stable solid phase is not necessarily closely packed. The density-functional theory applied to nonuniform classical liquids has been able to depict a wide range of physical properties of simple solid systems [14][23]. It is well known that the hard sphere model is used as the "reference" system, but to make the system more realistic, we need to include some attractive interactions. Accordingly, a considerable effort has gone into developing a free energy function describing nonuniform hard sphere systems and at present there exists a quite better functional approach for these systems. Unfortunately no equation of state is known which allow for hard disk and the hard sphere fluids in the close-packing limit [10]. A new problem appears when the density-functional theory is applied to less packed crystal structures such as the bbc lattice, which can be at most a metastable state of the system within the density functional approach. It should be noted that the density functional theory gives better results for higher densities of solid state matter [4] [9] [10]. Thus to study the fluid state, it seems useful to start from the solid state density functional.

The problem of the solid state formation has not yet been finally solved. From the point of the density functional theory, this means that there is no common density functional that describes the system of interacting particles at all densities from gas to solid state. Instead we have one density functional well adapted for gas phase and another one for solid or liquid phase. Another problem is that the system of interacting particles crystallizes after passing through many intermediate states such as a supercooled liquid. The description of a supercooled liquid state encounters serious difficulties both in computer modelling and in theoretical approach, when it remains the problem of reduction of possible states of the system in its statistical description. We always can describe criteria of instability in a system but we can not specify a final state. A considerable fraction of the elements, when compressed, undergo a first order phase transition from a disordered state (a gas, fluid, or liquid) to a face-centered cubic ( $\mathrm{fcc}$ ) or hexagonal close packed (hcp) crystal. Computer simulations as well as experiments show that this freezing transition is already apparent in the simple model of interacting particles [3] [5] [6] [7]. Theory can explain only the transition "afterwards". A complete theory of hard sphere freezing should therefore identify this property of the partition function that leads to observed symmetry breaking. Thus, it seems that one obtain better description when the final state is given a priori and the conditions under which this state exists are determined from the density functional theory. In this sense the description of simple model system of interacting spheres with a finite energy of contact repulsion and long-range attraction of a gravitational type is offered. It is known that particle systems with purely attractive gravitational interaction exhibit collapse, sometimes called a zero-order phase transition [8] [18]. If no short-range cutoff are introduced, then the discontinuous jump is infinite. This makes all normal noncollapsed states of the self-attractive system metastable with respect to such a collapse. On the other hand, if the same form of short-range cutoffs are introduced, the 
entropy and free energy jumps are finite. In this case, in the result of a collapse, the system goes into a nonsingular state with a dense core, the precise nature of which depends on the details of the short-range behavior of the potential. This model system of interacting particles can be used to describe the final state of the mutually gravitating particles with a firm nucleus or the colloidal particles in liquids and liquid crystals.

The second aspect of the solid state formation is that for systems with long-range attraction and short-range repulsion the system crystallizes passing through states with different packing (for example, cubic structure can transform to hexagonal). In the proposed approach we can find not only the criteria of formation of the particular crystal structure but we can also describe the transformations of the system from one packing to another. The phase transitions with change of packing due to the change of particle shape resulted from strong interparticle attraction (for example, to become a deformed spherical colloidal particle) are also can be described within the developed approach. It should be emphasized that the contact repulsion between the particles implies the use of the Fermi statistics even for classical particles and finite energy of contact repulsion that is because particles can not penetrate each other. This feature was not taken into account in the statistical description of phase transitions into a crystal state [24].

Let us consider a model system of particles on a lattice interacting via the following potential

$$
W\left(r-r^{\prime}\right)=-\frac{G m^{2}}{\left|r-r^{\prime}\right|}+U \theta\left(2 R_{0}-\left|r-r^{\prime}\right|\right)
$$

where the first term accounts for the long-range Newtonian attraction between particles and the second one corresponds to the short-range repulsion with $R_{0}$ being the particle radius, here $\theta$ is the Heaviside step function. Within the density-functional theory the free energy for this system can be written in the form [25] [26]

$$
F=\frac{1}{2} \sum_{r, r^{\prime}} W\left(r-r^{\prime}\right) f(r) f\left(r^{\prime}\right)+k T \sum_{r}[f(r) \ln f(r)-(1-f(r)) \ln (1-f(r))],
$$

where $f(r)$ is the one-particle distribution function (the probability to find a particle in point $r$ ) and the summation is over the lattice. We assume hard sphere model when each site of the lattice can be occupied by only one particle. This feature is accounted by Fermi type of the entropy part in (2).

By minimizing the free energy (2) we obtain some particles distribution $f(r)$ which corresponds to some equilibrium state of the system. Depending on the temperature and interaction strength the distribution can be homogeneous or inhomogeneous. If the equilibrium state is disordered then the one-particle distribution function is homogeneous, that is $f(r)=f_{0}$, where $f_{0}$ is the relative particle concentration. Any inhomogeneity in particles distribution can be described by the additional term $\Delta f(r)$ in the expression $f(\boldsymbol{r})=f_{0}+\Delta f(r)$ that in the Fourier space can be expressed as $f(r)=f_{0}+\sum \Delta f(q) \exp ($ iqr $)$, where the sum is now over reciprocal lattice vector $q$. In the density-functional theories the solid density is given 
a priori by a Gaussian parametrization $f()=(\pi / a)^{\frac{3}{2}} \sum_{R} \exp \left(-a|r-R|^{2}\right)$, where the sum is over the lattice vector $R$ and depends upon the particular crystal structure, and $a$ is the order parameter. The value of parameter $a$ being different for gas, liquid, and solid phases determines the phase of the system. In our case $f_{0}=N / N_{0}=\alpha v=6 v / \pi$, where $v$ is the packing factor. It should be noticed that the multiplier $\alpha$ is determined by the particular arrangement of atoms in a unit cell of the crystal. The value $\alpha=6 / \pi$ corresponds to a cubic cell of linear size equal to the particle's diameter. The small values of the order parameter $a$ correspond to the situation typical for solids, when atoms are strongly localized around the ideal lattice sites, and the free energy can be reduced to

$$
F\left(f_{0}\right)=\frac{1}{2} N^{2}\left(U v^{2}-\frac{3 W_{G} v^{\frac{1}{3}}}{2 N^{\frac{1}{3}}}\right)+k T\left[\ln \alpha v+\frac{1-\alpha v}{\alpha v} \ln (1-\alpha v)\right],
$$

where $W_{G}=G m^{2} / R_{0}$. Then the equation of state is

$$
P=-\left(\frac{\partial F}{\partial V}\right)_{T, N}=-\frac{\partial F}{\partial v} \frac{\partial v}{\partial V}
$$

In our case $-\frac{\partial V}{\partial V}=\frac{V}{V}$, thus (4) reduces to

$$
P V=\frac{\partial F}{\partial v} v=\frac{1}{2} N^{2}\left(U v-\frac{W_{G} v^{\frac{1}{3}}}{2 N^{\frac{1}{3}}}\right)-\frac{k T N}{\alpha v} \ln (1-\alpha v) .
$$

Note that $V$ is the whole system volume including the volume occupied by particles. In the limit of low packing factor, $v \ll 1$, we obtain the equation of state of lattice gas with interaction between particles:

$$
P V=k T N+\frac{1}{2} N^{2}\left(U v-\frac{W_{G} v^{\frac{1}{3}}}{2 N^{\frac{1}{3}}}\right)
$$

This equation is like a Van der Waals equation of state for hard spheres with the second part of this equation representing the interaction in the system. The formula (6) may serve as a rather good approximation for an equation of state for systems with a repulsive hard core and a newtonian attractive potentials. The thermodynamic properties of elastic hard-sphere systems depend on the temperature in a trivial manner. Two methods can be used to calculate the pressure in the system. The first is based on calculating the rate $h$ from which the pressure can be deduced by the formula $\frac{P V}{k T N}=1+\frac{h}{h_{0}}$, where $h_{0}=8 \sqrt{\pi\left\langle v^{2}\right\rangle / 3} R_{0}^{2} N(N-1) / V$ is the low-density collision rate for large packing factors of hard spheres gas, $\left\langle v^{2}\right\rangle$ is the mean square velocity and is proportional to $T$. The second method utilizes the following relation between the equation of state of hard spheres gas and the value of the radial distribution function $g(r)$ at $r=2 R_{0}: \frac{P V}{k T N}=1+4 v g\left(2 R_{0}\right)$. These two methods give close values but the 
second one requires some care because the radial distribution function varies rapidly in the vicinity of $r=2 R_{0}$ [11]. Another equation of state of the hard spheres gas, $P V=k T N \frac{1+v+v^{2}-v^{3}}{(1-v)^{3}}$, where a particle volume is excluded, has quit different form though asymptotically is similar to above ones. When $W_{G} /(2 U) \geq N^{1 / 3} v^{2 / 3}=N\left(R / R_{0}\right)^{2}$ and thus $R / R_{0} \leq N\left(W_{G} / 4 k T\right)$, where $R$ is the size of the system, the collapse in system is possible. The corresponding packing factor is $v=\left(4 k T / W_{G}\right)^{3} N^{-2}$. The condition of collapse satisfy to zero value of the pressure, this yields

$$
\frac{1}{2} N^{2}\left(U v-\frac{W_{G} v^{\frac{1}{3}}}{2 N^{\frac{1}{3}}}\right)-\frac{k T N}{\alpha v} \ln (1-\alpha v)=0
$$

In the case when $\alpha v \rightarrow 1$

$$
\alpha v=1-\exp \frac{1}{2 \alpha}\left(\frac{N U}{k T}-\frac{W_{G} \alpha^{\frac{1}{3}} N^{\frac{2}{3}}}{2 k T}\right) .
$$

Thus a collapse is possible when the mean distance between particles is less than $\frac{W_{G}}{2 U} R_{0}$.

To study the effect of inhomogeneity of particles distribution we substitute $f(\boldsymbol{r})=f_{0}+\Delta f(\boldsymbol{r})$ into (2) and make use of the long-wavelength expansion of the concentration. The result is

$\Delta F \equiv F-F\left(f_{0}\right)=\frac{N}{2 V} \sum_{\boldsymbol{q}}\left[-\frac{4 \pi Q^{2}}{q^{2}}\left(\delta_{i j}-\frac{q_{i} q_{j}}{q^{2}}\right)+U \frac{4 \pi}{3} R_{0}^{3}+\frac{k T}{\alpha v(1-\alpha v)}\right] \Delta f(\boldsymbol{q}) \Delta f(-\boldsymbol{q})$.

For the hexagonal packing we have [24]:

$$
q R_{0}=\left(\frac{3 W_{G}}{4 U}\right)^{\frac{1}{2}}\left(1+\frac{k T}{U \alpha v^{2}(1-\alpha v)}\right)^{-\frac{1}{2}},
$$

and when $k T \rightarrow 0$ this reduces to

$$
q R_{0}=\left(\frac{3 W_{G}}{4 U}\right)^{\frac{1}{2}}
$$

If $W_{G} / U>4 \pi^{2} / 3$ then the inhomogeneity length becomes smaller than the contact distance between particles: $2 \pi / q<2 R_{0}$. In this case the attraction obviously prevails repulsion resulting in deformation of a particle with a soft nucleus. Thus the form of a cell can change from the initial cubic cell to the hexagonal. The origin of such a transformation can be explained in the following way. An attraction between particles causes a collapse in the system. The particles begin to press upon one another that results in the deformation of their shapes. A final equilibrium state in such a system is when the system volume is equal to the total volume of all the particles. But this does not change the volume of a separate particle but only changes the form of the particle. For liquid particles it is possible to estimate pressure created by a gravitational 
attraction and to compare it to the Laplacian pressure in a separate liquid particle. We can estimate it from the condition $\frac{W_{G}}{\sigma R^{2}} \geq \frac{4}{m}$, where $\sigma$ is the surface energy and $m$ is the number of possible nearest neighbors. The number of nearest neighbors in the close packing $m=12$. When particles are deformed the distance between their centers must be less than $d$. Thus the condition $\frac{4 \pi^{2}}{3} \geq \frac{4}{m}$ corresponds to the condition of deformation for such a structural unit with a soft nucleus. If $k T \gg U \alpha v^{2}(1-\alpha v)$ then the formula (10) gives the period of particles distribution to be $\lambda=\pi d\left(\frac{4 k T}{3 W_{G} \alpha v^{2}(1-\alpha v)}\right)^{1 / 2}$. In this case the equilibrium structure of the system is determined by only the attractive and kinetic energy and is not influenced by the short-range repulsion. In a system of emulsion particles in a fluid media with the screened Coulomb-like interaction it is possible to observe structural phase transitions with change of the shape of the droplets.

Thus the behavior of interacting particles with long-range attraction and short-range repulsion can be described in all equilibrium phases [27]. Starting from a gas phase the attraction results in the condensation of the system. In the condensed matter phase with lowering the temperature the attraction induces transition into solid state with the packing factor of cubic structure. At some ratio of the attraction and repulsion intensities there can be a new structural transition with change of the packing factor. The cubic lattice transforms into hexagonal structure. Thus the form of the structure unit can change without change of its volume, for example, sphere can transform into some dodecahedron (or disk into hexagonal cell). The possible volume of the system will be equal to the sum of individual volumes of separate structural units. Thus even a collapse of the system produced by a strong attraction between particles will be finished by the creation of a new structure with the structural factor up to unit, and this state is final for systems with strong long-range attraction and short-range repulsion of a soft type.

This research was supported by the Basic Science Research Program through the National Research Foundation of Korea (NRF) funded by the Ministry of Education, Science and Technology (NRF-2014R1A1A2058029), and supported by Brain Pool Program through the Korean Federation of Science and Technology Societies (KOFST) funded by the Ministry of Science, ICT and Future Planning (161S-1-3-1617).

\section{References}

[1] Denton, A.R., Ashcroft, N.W. and Curtin, W.A. (1995) Physical Review E, 51, 65. https://doi.org/10.1103/PhysRevE.51.65

[2] Tarazona, P. (1985) Physical Review A, 31, 2672. https://doi.org/10.1103/PhysRevA.31.2672 Tarazona, P. (2000) Physical Review Letters, 84, 694. https://doi.org/10.1103/PhysRevLett.84.694 Tarazona, P. and Rosenfeld, Y. (1997) Physical Review E, 55, R4873. https://doi.org/10.1103/PhysRevE.55.R4873

[3] Rosenfeld, Y., Schmidt, M., Lowen, H. and Tarazona, P. (1997) Physical Review E, 55, 4245. 
https://doi.org/10.1103/PhysRevE.55.4245

Rosenfeld, Y. (1989) Physical Review Letters, 63, 980.

https://doi.org/10.1103/PhysRevLett.63.980

[4] Rascon, C., Mederos, L. and Navascues, G. (1996) Physical Review E, 53, 5698.

https://doi.org/10.1103/PhysRevE.53.5698

[5] Laird, B.B. and Kroll, D.M. (1980) Physical Review A, 42, 4810.

https://doi.org/10.1103/PhysRevA.42.4810

[6] Kim, S.C. (1997) The Journal of Chemical Physics, 106, 1146.

[7] Keget, W.K., Retas, H. and Lekkerkerker, H.N.W. (1999) Physical Review Letters, 83, 5298. https://doi.org/10.1103/PhysRevLett.83.5298

[8] Ispolatov, I. and Cohen, E.G.D. (2001) Physical Review Letters, 87, 210601. https://doi.org/10.1103/PhysRevLett.87.210601

[9] Eu, B.C. and Rah, K. (2001) Physical Review E, 63, 031203. https://doi.org/10.1103/PhysRevE.63.031203

[10] Wang, X.Z. (2002) Physical Review E, 66, 031203. https://doi.org/10.1103/PhysRevE.66.031203

[11] Richard, P., Oger, L., Troadee, J.-P. and Gervois, A. (1999) Physical Review E, 60, 45514558. https://doi.org/10.1103/PhysRevE.60.4551

[12] Caccamo, C., Pellicane, G., Costa, D., Pini, D. and Stell, G. (1999) Physical Review E, 60, 5533-5543. https://doi.org/10.1103/PhysRevE.60.5533

[13] Wang, D. and Gast, A. (1999) Physical Review E, 59, 3964-3969. https://doi.org/10.1103/PhysRevE.59.3964 Rascon C., Mederos, L. and Navascues, G. (1996) Physical Review E, 53, 5698-5703. https://doi.org/10.1103/PhysRevE.53.5698

[14] Hess, S. and Kroger, M. (2001) Physical Review E, 64, Article ID: 011201. https://doi.org/10.1103/PhysRevE.64.011201

[15] Edgal, U.F. and Huber, D.L. (1998) Journal of Chemical Physics, 108, 1578-1586. https://doi.org/10.1063/1.475529

[16] Likos, C.N., Lang, A., Watzlawek, M. and Lowen, H. (2001) Physical Review E, 63, Article ID: 031206. https://doi.org/10.1103/PhysRevE.63.031206

[17] Dixit, N.M. and Zukoski, C.F. (2001) Physical Review E, 64, Article ID: 041604. https://doi.org/10.1103/PhysRevE.64.041604

[18] Bergenholtz, J. and Fuchs, M. (1999) Physical Review E, 59, 5706-5715. https://doi.org/10.1103/PhysRevE.61.5218

[19] Groh, B. (2000) Physical Review E, 61, 5218-5222. https://doi.org/10.1103/PhysRevE.61.5218

[20] Kobelev, V. and Kolomeisku, B. (2002) Journal of Chemical Physics, 116, 7589-7598. https://doi.org/10.1063/1.1464827

[21] Anta, J.A. and Lago, S. (2002) Journal of Chemical Physics, 116, 10514-10522. https://doi.org/10.1063/1.1479140

[22] Herrera, J.H., Ruiz-Estrada, H. and Blum, L. (1996) Journal of Chemical Physics, 104, 6327-6329. https://doi.org/10.1063/1.471293

[23] Babin, V., Ciach, A. and Tasinkevych, M. (2001) Journal of Chemical Physics, 114, 95859592. https://doi.org/10.1063/1.1370082

[24] Kosterliz, J.M. and Thouless, D.J. (1973) Journal of Physics C: Solid State Physics, 6, 1181- 
1203. https://doi.org/10.1088/0022-3719/6/7/010

Stranburg, K.J. (1988) Reviews of Modern Physics, 60, 161-207.

https://doi.org/10.1103/RevModPhys.60.161

[25] Kleinert, H. (1989) Gauge Fields in Condensed Matter. World Scientific, Singapore. https://doi.org/10.1142/0356

[26] Khachaturyan, A.J. (1983) Theory of Structural Transformations in Solids. Wiley, New York.

[27] Lev, B.I. and Zhugajevich, Y. (1998) Physical Review E, 57, 6460-6469.

https://doi.org/10.1103/PhysRevE.57.6460

Submit or recommend next manuscript to SCIRP and we will provide best service for you:

Accepting pre-submission inquiries through Email, Facebook, LinkedIn, Twitter, etc.

A wide selection of journals (inclusive of 9 subjects, more than 200 journals)

Providing 24-hour high-quality service

User-friendly online submission system

Fair and swift peer-review system

Efficient typesetting and proofreading procedure

Display of the result of downloads and visits, as well as the number of cited articles

Maximum dissemination of your research work

Submit your manuscript at: http://papersubmission.scirp.org/

Or contact jmp@scirp.org 